\title{
The accuracy and dosimetric analysis of 3D-printing non-coplanar template-assisted iodine-125 seed implantation for recurrent chest wall cancer
}

\author{
Weijuan Jiang, MD, Yi Chen, MD, Zhe Ji, MD, Yuliang Jiang, MM, Bin Qiu, MD, Haitao Sun, MM, Prof. Junjie Wang, MD \\ Department of Radiation Oncology, Peking University Third Hospital, Beijing, China
}

\begin{abstract}
Purpose: To investigate the accuracy and dosimetry of 3D-printing non-coplanar template (3D-PNCT)-assisted computed tomography (CT)-guided iodine-125 seed implantation $\left({ }^{125} \mathrm{I}-\mathrm{SI}\right)$ for recurrent chest wall cancer (rCWC).

Material and methods: This was a retrospective study of 19 patients with 22 rCWC treated with 3D-PNCT-assisted CT-guided ${ }^{125} \mathrm{I}-\mathrm{SI}$, from Mar 2017 to Mar 2020 in our institute.

Results: Mean needle entrance deviation was $4.50 \pm 2.70 \mathrm{~mm}$, mean angular deviation was $3.40 \pm 3.10$ degrees, and mean depth deviation was $5.20 \pm 5.20 \mathrm{~mm}$. No significant difference was found for dosimetric parameters (except conformity index) between pre-plan and post-plan; $\mathrm{D}_{90}, \mathrm{D}_{100}, \mathrm{~V}_{100}, \mathrm{~V}_{150}$, and $\mathrm{V}_{200}$ were $157.74 \pm 24.23$ and $151.71 \pm 33.62$ $(p=0.228), 85.36 \pm 34.09$ and $70.46 \pm 23.48(p=0.067), 0.93 \pm 0.04$ and $0.90 \pm 0.07(p=0.068), 0.64 \pm 0.16$ and $0.64 \pm 0.16$ $(p=0.984)$, and $0.35 \pm 0.17$ and $0.37 \pm 0.18(p=0.382)$ for pre-plan and post-plan, respectively. Conformity index, external index, and homogeneity index were $0.57 \pm 0.16$ and $0.52 \pm 0.15(p=0.007), 0.73 \pm 0.55$ and $0.79 \pm 0.53(p=0.096)$, and $0.31 \pm 0.15$ and $0.30 \pm 0.14(p=0.504)$ for pre-plan and post-plan, respectively. Median follow-up time was 8 months (range, 3-30 months). Complete response was observed in 4/22 (18.1\%), partial response in 13/22 (59.1\%), stable disease in $4 / 22(18.1 \%)$, and progression disease in $1 / 22(4.5 \%)$ of the cancers. Among patients with pain before ${ }^{125}$ I-SI, pain relief rate was $87.5 \%(7 / 8)$. No peri-operative complications of more than grade 2 were observed.

Conclusions: 3D-PNCT-assisted CT-guided ${ }^{125} \mathrm{I}$-SI may be safe and feasible as palliative therapy for non-surgical candidates and painful patients with rCWC.

J Contemp Brachytherapy 2021; 13, 3: 273-279 DOI: https://doi.org/10.5114/jcb.2021.106250
\end{abstract}

Key words: chest wall cancer, 3D printing, ${ }^{125} \mathrm{I}$, seed implantation, brachytherapy.

\section{Purpose}

Chest wall cancer (CWC) may derive from a variety of cancers, including breast cancer, mesothelioma, sarcoma, lung cancer, and esophageal cancer [1-3]. Recurrent CWC (rCWC) is complicated with limited salvage therapy after failure of surgery/external beam radiotherapy (EBRT) [3-5]. Chemotherapy with or without local therapy is a palliative option. Salvage surgical resection/EBRT may be used in selected patients to improve local control depending on the number and extensions of the lesions. The extent of resection significantly influences survival, with satisfying clinical outcome absent in many patients who can only receive limited resection, because extensive resection carry a high-risk of disfiguring [3]. EBRT is an alternative option for patients without prior EBRT; however, it is hard to deliver a high-dose to the lesions owing to the movement and irregular nature of rCWC. It is difficult to use for patients with prior EBRT with a high-risk of radiation-related toxicities, such as chest fibrosis, pneumonia, and rib fracture [5].
Computed tomography (CT)-guided iodine-125 seed implantation ( $\left.{ }^{125} \mathrm{I}-\mathrm{SI}\right)$ is proved to be safe and effective when applied in head and neck, thoracic, abdomen, retroperitoneal, and vertebral cancers, especially for locally advanced or recurrent solid cancers after EBRT [6-12]. ${ }^{125} \mathrm{I}-\mathrm{SI}$ may be applied as a salvage therapy for $\mathrm{rCWC}$ alone or as a boost to EBRT, due to rapid dose falling-off and sparing of surrounding organs, while the chest wall is irregular and it may be difficult to perform CT-guided ${ }^{125} \mathrm{I}$-SI in patients with rCWC. Organ motion and non-uniformity of the organ may interference with the needle insertion during seed implantation and lead to a sub-optimal distribution of seeds, partly due to the ribs interference [10].

The main challenge is how to deliver the ${ }^{125}$ I seeds into the target volume accurately according to pre-plan. 3D-printing non-co-planar template (3D-PNCT) is helpful for high quality performance of CT-guided ${ }^{125} \mathrm{I}$-SI $[7,8,12-14]$. The safety, accuracy, and feasibility of 3D-PNCT application in CT-guided ${ }^{125} \mathrm{I}$-SI for cancers
Address for correspondence: Prof. Junjie Wang, Peking University Third Hospital, 49 North Garden Road, Haidian District, Beijing, 100191, P. R. China, phone: +86-13701076310, fax: +86-10-62017700,

$\bowtie$ e-mail: junjiewang@pku.edu.cn
Received: 14.01 .2021

Accepted: 20.03 .2021

Published: 18.05 .2021 
raised in complex locations, such as the head and neck, has been explored and reported [7, 8, 10, 12-15]. However, the literature on the accuracy and dosimetric analysis of 3D-PNCT-assisted ${ }^{125} \mathrm{I}-\mathrm{SI}$ for rCWC is scarce. Therefore, the present retrospective study analyzed the accuracy and dosimetric parameters of 3D-PNCT-assisted CT-guided ${ }^{125} \mathrm{I}$-SI in patients with $\mathrm{rCWC}$.

\section{Material and methods}

\section{Study design and population}

In the retrospective study, 19 consecutive patients with 22 rCWC lesions treated with ${ }^{125}$ I-SI between Mar 2017 and Mar 2020 were included. Among them, 14 patients were previously treated with EBRT once, 2 patients were treated twice, and the remaining 3 patients refused EBRT (Table 1). The inclusion criteria of ${ }^{125} \mathrm{I}$-SI were as follows: 1) age 18-80 years, Karnofsky performance status (KPS) score more than 70 ; 2) pathologically or radiolog-

Table 1. Characteristics of the 19 patients

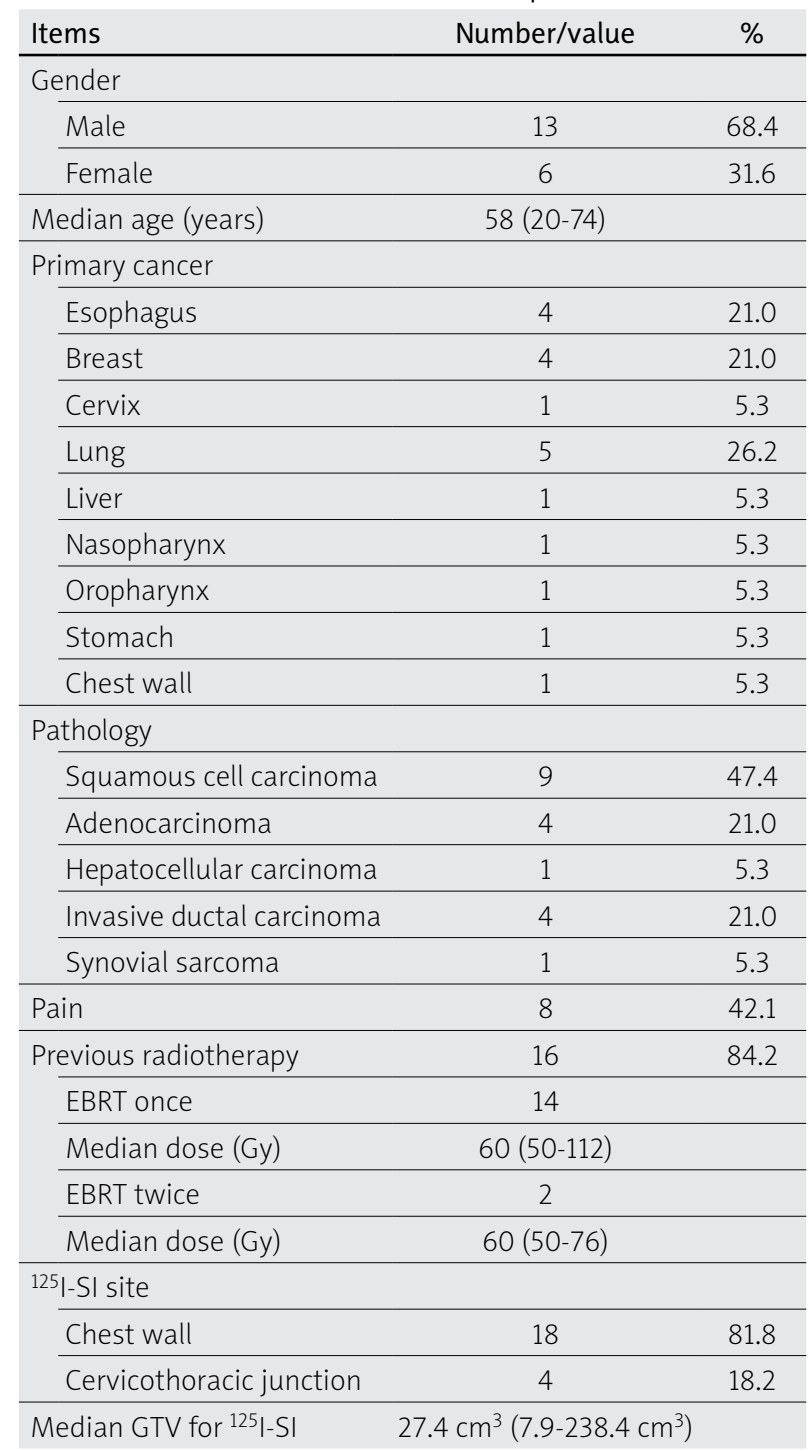

EBRT - external beam radiotherapy, ${ }^{125} /$-SI - $125 /$ seed implantation, GTV - gross tumor volume ically diagnosed rCWC (defined as primary/secondary cancers raised from the chest/cervicothoracic junction); 3) the diameter of the lesion less than $7 \mathrm{~cm}$, the number of lesions less than 3 [8]; 4) expected survival time more than 3 months; and 5) intolerant/refused to surgery/ EBRT. Contraindications were as follows: 1 ) severe organ function disorder; 2) coagulation dysfunction; 3) recent active infection; 4) mental illness; and 5) lesions invasive to the skin (avoiding refractory skin ulcer that may be caused by $\left.{ }^{125} \mathrm{I}-\mathrm{SI}\right)$.

The number of needles and seeds, deviation of needles entrance, angle and depth for pre-plan, and the intra-operative real-time plan were measured on brachytherapy treatment planning system (B-TPS) according to a previous report [13]. The dosimetric parameters including $\mathrm{D}_{90}, \mathrm{D}_{100}, \mathrm{~V}_{100}, \mathrm{~V}_{150}, \mathrm{~V}_{200}$, conformity index $(\mathrm{CI})$, external index (EI), and homogeneity index (HI) were compared between pre-plan and post-plan. The dosimetric parameters were defined as doses delivered to $90 \%\left(D_{90}\right)$ and $100 \%\left(D_{100}\right)$ of gross tumor volume $(\mathrm{GTV})$ and percentage of GTV receiving $100 \%\left(\mathrm{~V}_{100}\right), 150 \%\left(\mathrm{~V}_{150}\right)$, and $200 \%\left(\mathrm{~V}_{200}\right)$ of the prescription dose. The peri-operative adverse events were evaluated during 1 month after ${ }^{125} \mathrm{I}$ SI according to the Radiation Therapy Oncology Group (RTOG) and European Organization for Research and Treatment of Cancer (EORTC) criteria [16]. The cancer response and patient survival were recorded. Pain was routinely assessed using a numerical rating scale (NRS), which was categorized into five grades: 0 for no pain, 1-3 for mild pain, 4-6 for moderate pain, 7-9 for severe pain, and 10 for unbearable pain [17]. The NRS score before ${ }^{125} \mathrm{I}-\mathrm{SI}$ was compared with that of 1 month after ${ }^{125} \mathrm{I}-\mathrm{SI}$.

\section{Pre-plan}

The patients were immobilized with a vacuum pad in a suitable position and underwent an enhanced CT (Brilliance, Philips Inc., The Netherlands) scan with a $5 \mathrm{~mm}$ slice thickness 1-2 days before ${ }^{125} \mathrm{I}$-SI. The position lines were marked on the patient skin according to the tumor's central location. CT simulation images were transferred into B-TPS (Beijing Aeronautics and Astronautics University and Beijing Astro Technology Co., Ltd.). Both GTV and organs at risks (OARs) were defined and delineated. Clinical tumor volume (CTV) was defined by expanding 5-6 $\mathrm{mm}$ in 3D directions from GTV. The median prescription dose was $120 \mathrm{~Gy}$ (range, 110-160 Gy), and the median activity of ${ }^{125} \mathrm{I}$ seed (the length $4.5 \mathrm{~mm}$, diameter $0.8 \mathrm{~mm}$, and half-life 59.4 days; Beijing Atom High Tech Pharmaceutical Company Inc., China) was $0.55 \mathrm{mCi}$ (range, 0.40-0.75 mCi). Dosimetric optimization was conducted and the D90 was optimized as close as to the prescription dose, whereas the doses of OARs were kept as low as possible.

\section{D-PNCT design and printing}

The number of needles and seeds, needles pathway, and seeds distribution were designed and simulated on B-TPS. The dose conformity of CTV was optimized while sparing doses to surrounding normal tissues. OARs, such as blood vessels, bony structure, and nerves, were avoid- 

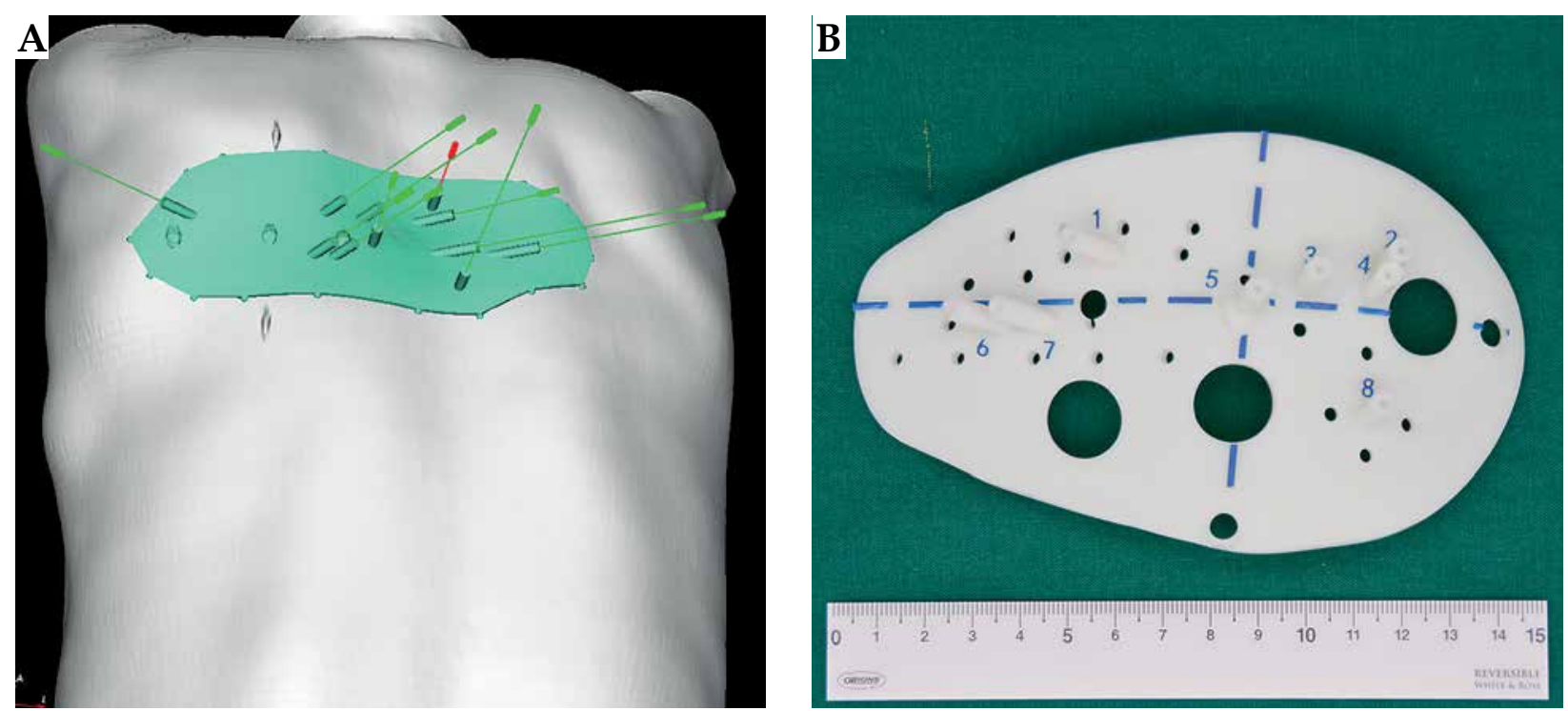

Fig. 1. A) Digital modeling of individualized 3D-printing non-coplanar templates (3DPNCT); B) 3D-PNCT included anatomical information of the target area, needles pathway and entrance, position lines, etc.

ed and kept away from the needle pathway usually with a safety distance of $0.5 \mathrm{~cm}$. The data was imported to Magics 19.01 (Materialise, Belgium) for digital modeling of individualized 3D-PNCT (Figure 1A). Then, 3D-PNCT was printed using a 3D light-cured rapid-forming printer (RS6000, Shanghai Liantaiv 3D Technology Company Inc., China). The 3D-PNCT consisted of anatomical information of the target area, needle pathway and entrance, and position lines, etc. (Figure 1B). Additional 2-3 holes for anchoring needles were added to fix the 3D-PNCT to the targeted area of the patients' body.

\section{${ }^{125} \mathrm{I}-\mathrm{SI}$ procedure}

The ${ }^{125}$ I-SI procedure has been described in our previous published literature [11]. In brief, the procedure was as follows: 1) Patients were immobilized with a vacuum pad. Local anesthesia was carried out. The 3D-PNCT was aligned to the target area of the patients' bodies according to the laser line marks and position lines (Figure 2). Two or 3 anchoring needles were inserted with 2-3 cm in depth to fix the 3D-PNCT; 2) CT scan was performed to check the anchoring needles position and angles; 3) Adjustment was made when the anchoring needles position was mismatched to pre-plan; 4) The seed needles were then all inserted according to pre-plan, once the anchoring needles were deemed in position; 5) CT scan was performed and adjustment was made when the position of the needle was mismatched to pre-plan, until all the needles were in position. Intra-operative real-time plan was created and conducted when necessary, by adding or dislodging the number of needles/seeds; 6 ) ${ }^{125}$ I seeds were delivered and implanted with an applicator (Mick 200-TPV Applicator, Mick Radio-Nuclear Inc., USA) in a regressive manner; 7) CT scan was performed again to verify the actual distribution of ${ }^{125}$ I seeds, and the images were transferred into B-TPS for a post-plan evaluation. The following data of post-plan were recorded: $D_{90}, D_{100}$,
$\mathrm{V}_{100}, \mathrm{~V}_{150}$, and $\mathrm{V}_{200}$. All procedures followed the recommendations of the International Commission on Radiological Protection (Figure 3A-C).

\section{Follow-up}

The patients were discharged 1-2 days after the ${ }^{125}$ I-SI procedure; follow-up assessments were routinely performed at 3, 6, 9, and 12 months after ${ }^{125}$ I-SI and then every 6 months thereafter until disease progression or patients' death. Follow-up assessments were conducted during regular outpatient visits (9 patients) or telephone

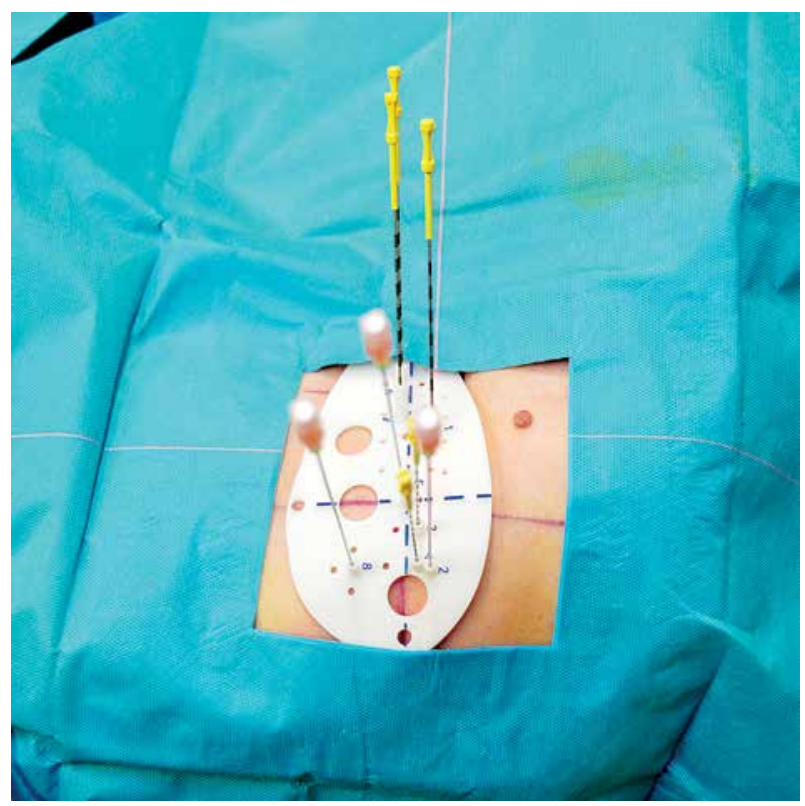

Fig. 2. 3D-printing non-coplanar templates (3D-PNCT) was aligned to target area of the patient's body according to the laser lines marks and position lines. Two or three anchoring needles (pink) and seed needles (yellow) were inserted 

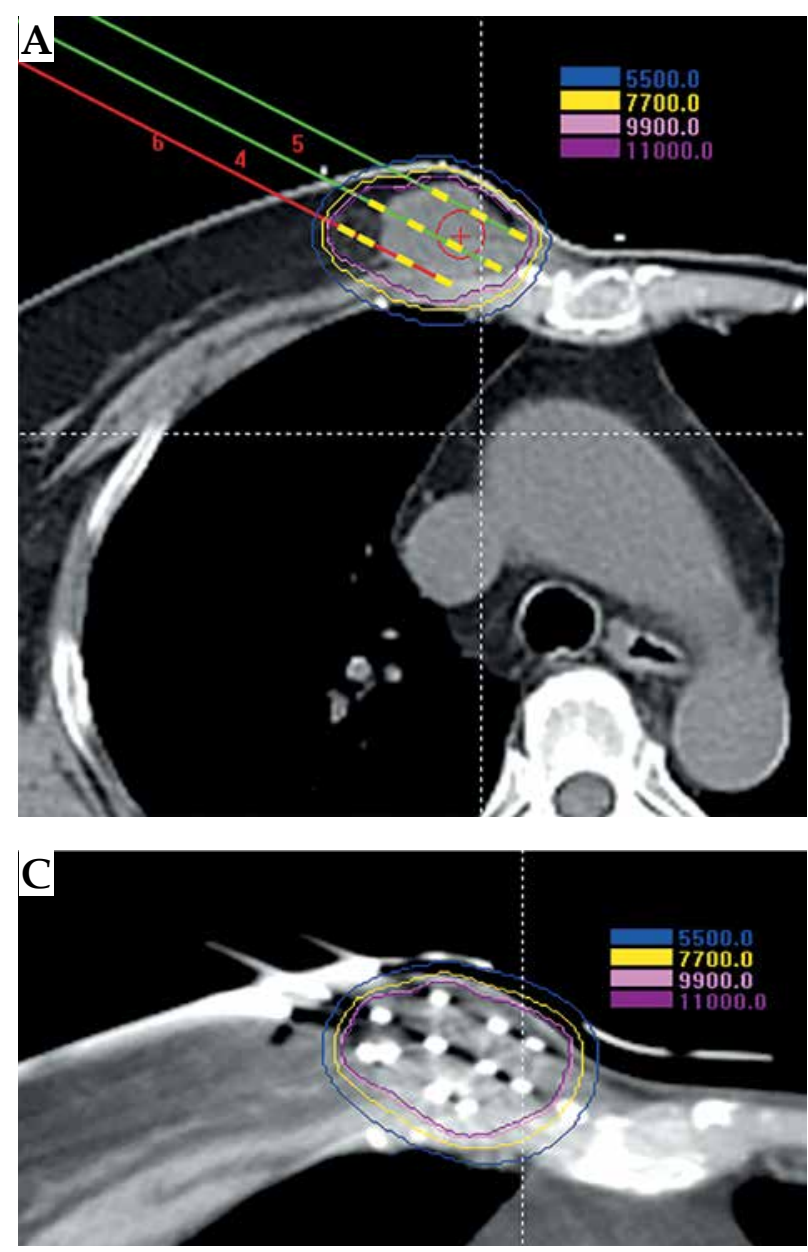

interviews (10 patients). CT scans/MRI examinations were used to evaluate the tumor response during each outpatient visit. Modified response evaluation criteria in solid tumors (mRECIST) version 1.1 criteria were used to assess local tumor response in 1 month after ${ }^{125}$ I-SI, and included complete response (CR), partial response (PR), stable disease (SD), and progressive disease (PD) [18].

\section{Statistical analysis}

Statistical analysis was performed using SPSS version 25.0 (IBM Corp, Armonk, NY, USA). Paired sample $t$-test was used to compare mean of pre-operative and postoperative dosimetric parameters. Bland-Altman method was applied to analyze the factors of pre-operative and post-operative dosimetric parameters, using MedCalc v.15.2.2 software. $P<0.05$ was considered statistically significant.

\section{Results}

The number of needles was $17.19 \pm 9.32$ and 17.29 \pm 9.22 per patient for pre-plan and post-plan, respectively $(p=0.629)$. The number of ${ }^{125} \mathrm{I}$ seeds was $74.14 \pm 43.64$ and $79.00 \pm 48.62$ per patient for pre-plan and post-plan, respectively $(p=0.444)$. The mean needle entrance deviation was $4.50 \pm 2.70 \mathrm{~mm}$, the mean needle angular devi-

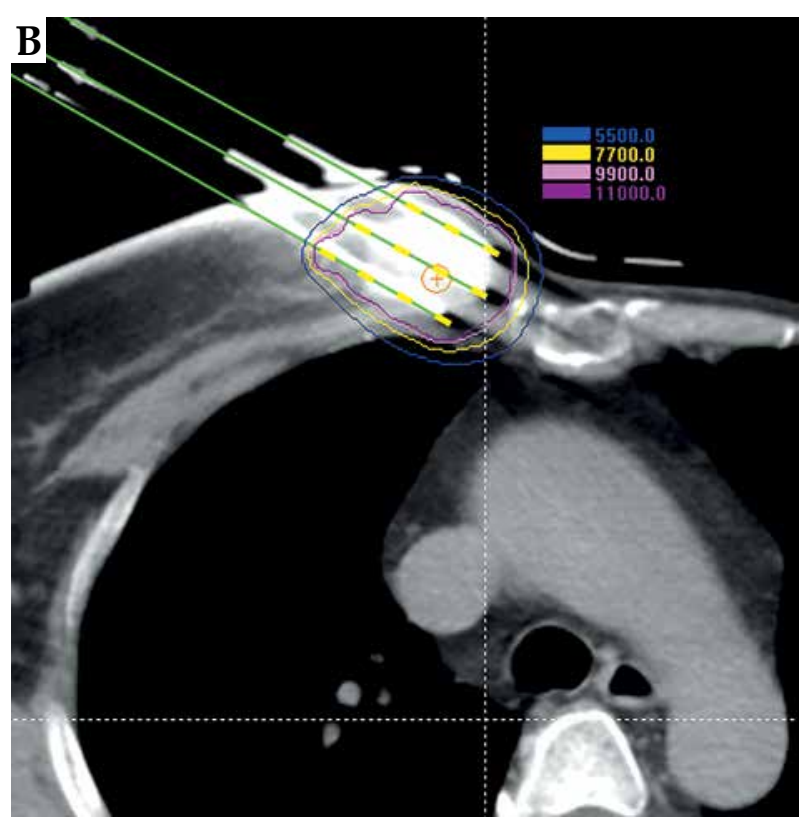

Fig. 3. A) Pre-plan, B) intra-operative, and C) post-plan validations. The color lines represent different isodose as shown in the graphical representation (blue, 5500 cGy; yellow, 7700 cGy; pink, 9900 cGy; brownness, 11000 cGy)

ation was $3.40 \pm 3.10$ degrees, and the mean depth deviation was $5.20 \pm 5.20 \mathrm{~mm}$.

No significant difference was found for dosimetric parameters (except $\mathrm{CI}$ ) between pre-plan and postplan (Table 2). The $\mathrm{D}_{90}, \mathrm{D}_{100}, \mathrm{~V}_{100}, \mathrm{~V}_{150}$, and $\mathrm{V}_{200}$ were $157.74 \pm 24.23$ and $151.71 \pm 33.62(p=0.228), 85.36 \pm 34.09$ and $70.46 \pm 23.48(p=0.067), 0.93 \pm 0.04$ and $0.90 \pm 0.07$ $(p=0.068), 0.64 \pm 0.16$ and $0.64 \pm 0.16(p=0.984)$, and $0.35 \pm 0.17$ and $0.37 \pm 0.18(p=0.382)$ for pre-plan and postplan, respectively. The CI, EI, and HI were $0.57 \pm 0.16$ and $0.52 \pm 0.15(p=0.007), 0.73 \pm 0.55$ and $0.79 \pm 0.53(p=0.096)$, and $0.31 \pm 0.15$ and $0.30 \pm 0.14(p=0.504)$ for pre-plan and post-plan, respectively.

The median follow-up time was 8 months (range, 3-30 months). The CR was observed in 4 of 22 (18.1\%), PR in 13 of $22(59.1 \%)$, SD in 4 of $22(18.1 \%)$, and PD in 1 of $22(4.5 \%)$ of the cancers. Among the 8 patients with pain before ${ }^{125}$ I-SI (5 patients with moderate pain and 3 patients with mild pain), the pain relief rate was $87.5 \%$ $(7 / 8)$. Complete relief was observed after ${ }^{125}$ I-SI in 2 patients with moderate and 1 patient with mild pain; partial remission was observed after ${ }^{125} \mathrm{I}-\mathrm{SI}$ in 3 patients with moderate pain, 1 patient with mild pain (Table 3 ). Only 1 patient suffered from mild pain during ${ }^{125} \mathrm{I}$-SI and the pain was immediately relieved after the ${ }^{125}$ I-SI procedure. No peri-operative complications (e.g., pneumothorax, 
Table 2. Dosimetric parameters of ${ }^{125}$ / seeds implantation (mean \pm SD)

\begin{tabular}{lcccccc} 
& Pre-plan & Intra-operative & Post-plan & $p^{*}$ & $p^{\star *}$ & $p^{\star \star *}$ \\
\hline Number of needles & $17.19 \pm 9.32$ & $17.29 \pm 9.22$ & $17.29 \pm 9.22$ & 0.629 & 0.629 & 0.999 \\
\hline Number of seeds & $74.14 \pm 43.64$ & $77.52 \pm 45.08$ & $79.00 \pm 48.62$ & 0.061 & 0.444 & 0.721 \\
\hline $\mathrm{D}_{90}$ & $157.74 \pm 24.23$ & $155.01 \pm 27.94$ & $151.71 \pm 33.62$ & 0.287 & 0.228 & 0.432 \\
\hline $\mathrm{D}_{100}$ & $85.36 \pm 34.09$ & $74.74 \pm 30.39$ & $70.46 \pm 23.48$ & 0.164 & 0.067 & 0.466 \\
\hline $\mathrm{V}_{100}$ & $0.93 \pm 0.04$ & $0.92 \pm 0.04$ & $0.90 \pm 0.07$ & 0.150 & 0.068 & 0.110 \\
\hline $\mathrm{V}_{150}$ & $0.64 \pm 0.16$ & $0.64 \pm 0.19$ & $0.64 \pm 0.16$ & 0.845 & 0.984 & 0.871 \\
\hline $\mathrm{V}_{200}$ & $0.35 \pm 0.17$ & $0.37 \pm 0.17$ & $0.37 \pm 0.18$ & 0.151 & 0.382 & 0.957 \\
\hline $\mathrm{Cl}$ & $0.57 \pm 0.16$ & $0.53 \pm 0.16$ & $0.52 \pm 0.15$ & 0.006 & 0.007 & 0.175 \\
\hline $\mathrm{El}$ & $0.73 \pm 0.55$ & $0.81 \pm 0.59$ & $0.79 \pm 0.53$ & 0.040 & 0.096 & 0.731 \\
\hline $\mathrm{HI}$ & $0.31 \pm 0.15$ & $0.29 \pm 0.14$ & $0.30 \pm 0.14$ & 0.074 & 0.504 & 0.560
\end{tabular}

$D_{90} / D_{100}$ - prescribed dose delivered to $90 \% / 100 \%$ of the gross tumor volume, $V_{100}-V_{150}$, and $V_{200}$ - gross tumor volume receiving $100 \%$, $150 \%$, and $200 \%$ of the prescribed dose, respectively, $\mathrm{Cl}$-conformation index, El-external volume index, $\mathrm{HI}$ - homogeneity index, ${ }^{*} p-p r e-p l a n$ vs. intra-operative, ${ }^{* *} p-p r e-p l a n$ vs. postplan, ${ }^{* * *} p$-intra-operative vs. post-plan

hematemesis, fever, and infection) of more than grade 2 were observed.

\section{Discussion}

Recurrent chest wall cancer tends to invade the skin, rib, or nerves inducing pain, which impairs patients' quality of life [19-21]. The recurrent lymph node metastasis at the inlet of thoracic invade the tracheal or artery inducing asphyxia and bleeding. The 3D-PNCT is suitable for patients with cancers located in the chest wall or cervicothoracic junction, and the advantages of ${ }^{125} \mathrm{I}$-SI are as follows: 1) the lesion is relatively superficial and immobilized; 2) the affluent bony structures facilitate 3D-PNCT fixation.

${ }^{125}$ I-SI as an adjuvant therapy appears to be a feasible modality, and patients recover and return to daily life soon after the treatment due to minimally invasive nature of the ${ }^{125} \mathrm{I}$-SI procedure. With the CT-guidance, ${ }^{125} \mathrm{I}$-SI is potentially able to deliver high doses to the target while sparing the surrounding normal tissues, with rapid dose falling-off of accurately implanted seeds. However, the challenge of ${ }^{125} \mathrm{I}-\mathrm{SI}$ is how to deliver the radioactive seeds into the targets accurately according to pre-plan in a complex anatomic structures, such as the head and neck, chest wall, retroperitoneal area, and pelvis. The dosimetric parameters of post-plan may not meet pre-plan requirements when the seeds are implanted inaccurately, and the outcomes may be compromised. Wan et al. [22] reported ${ }^{125} \mathrm{I}$-SI for prostate with an 18-gauge needle to test the accuracy between needle deflection and insertion depth; a needle bending occurred when the needle was inserted deeply. The results showed that the needle tended to diverge and/or bend from its pre-planned trajectory due to asymmetric resistance on the beveled tip of the needle. Rotation of the needle was a solution for decreasing the targeting error. Mean needle targeting errors were 0.8 and $1.2 \mathrm{~mm}$ and seeds implanted errors were 0.9 and $1.5 \mathrm{~mm}$ for needle rotation and non-needle rotation methods, respectively [22].
Table 3. Numerical rating scale (NRS) for the 8 painful patients

\begin{tabular}{lcc} 
Patient no. & Pre-seed implantation & $\begin{array}{c}\text { Post-seed implanta- } \\
\text { tion }\end{array}$ \\
\hline 1 & 2 & 2 \\
\hline 2 & 4 & 1 \\
\hline 3 & 4 & 0 \\
\hline 4 & 4 & 1 \\
\hline 5 & 3 & 0 \\
\hline 6 & 3 & 1 \\
\hline 7 & 4 & 2 \\
\hline 8 & 4 & 0
\end{tabular}

The 3D-PNCT was also a useful way to improve the accuracy of ${ }^{125} \mathrm{I}$-SI obtaining a high-dose in target volume with few needles and complications for advanced or recurrent cancers, particularly for cancers located in complex anatomies [23-25]. Huang et al. [13] reported the accuracy of 3D printing individual templates for ${ }^{125} \mathrm{I}-\mathrm{SI}$ in head and neck cancer. Mean needle entrance deviation for all 619 needles was $1.18 \pm 0.81 \mathrm{~mm}$, varying from 0.857 \pm 0.545 to $1.930 \pm 0.843 \mathrm{~mm}$ at different sites. Mean needle angular deviation was $2.08 \pm 1.07$ degrees, varying from $1.85 \pm 0.93$ to $2.73 \pm 1.18$ degrees at different sites. Here, the mean needle entrance deviation was $4.50 \pm 2.70 \mathrm{~mm}$, the mean angular deviation was $3.40 \pm 3.10$ degrees $(p=0.377)$, and the mean depth deviation of the needles was $5.20 \pm 5.20$. The deviations were all tended to be larger than that reported by Huang et al. in the head and neck, which may partly be due to 1) the breath and chest wall motion during ${ }^{125}$ I-SI, 2) dense OARs, such as blood vessels, trachea, nerves, etc., 3) 3D-PNCT for the chest was larger than that of the head and neck, which may induce slight 3D-PNCT tortuosity and changes of the needles pathway. The mean post-operative $D_{90}$ and $D_{100}$ were 151.71 Gy and 70.46 Gy, respectively. The differences were not significant between the pre-plan and post-plan, 
which means that the dosimetric data may fulfill the preplan requirements during 3D-PNCT-assisted CT-guided ${ }^{125} \mathrm{I}-\mathrm{SI}$. The CI of the post-plan was lower than the preplan $\mathrm{CI}$, because seeds position may differ from the preplan. Besides, with the assistance of 3D-PNCT during CT-guided interventions, the procedure may be simplified and the operator can be skilled after a relatively short learning time [26].

The present study had several limitations. First, this was a retrospective study with relatively small number of patients, which may lead to potential bias. Second, the absence of control group has also compromised the interpretation of the results. Furthermore, the group of patients was completely heterogeneous concerning tumor sites and pathologies. Finally, the data were obtained from image fusion on B-TPS, which may suffer from potential fusion errors.

\section{Conclusions}

3D-PNCT-assisted CT-guided ${ }^{125} \mathrm{I}$-SI may be safe and feasible as palliative therapy for non-surgical candidates and painful patients with rCWC.

\section{Funding}

National Key Research and Development Plan of China (grant no. 2019YFB1311300) to JJ W supported the implementation (e.g., labor cost and data collection) and publication of the project.

\section{Ethics approval}

This retrospective study was approved by the Peking University Third Hospital Medical Science Research Ethics Committee and the requirement to obtain written informed consent was waived.

\section{Availability of data and materials}

The datasets used and/or analyzed during the current study are available on request from the corresponding author.

\section{Acknowledgments}

The authors extend sincere gratitude to Dr. Yi Shi and Peng Zhen for the needle deviation measurement and data collection.

\section{Disclosure}

The authors report no conflict of interest.

\section{Reference}

1. Sabanathan S, Shah R, Mearns AJ. Surgical treatment of primary malignant chest wall tumors. Eur J Cardiothorac Surg 1997; 11: 1011-1016.

2. Salminen SH, Sampo MM, Bohling TO et al. Radiation-associated sarcoma after breast cancer in a nationwide population: increasing risk of angiosarcoma. Cancer Med 2018; 7: 4825-4835.
3. Clarke M, Collins R, Darby S et al. Effects of radiotherapy and of differences in the extent of surgery for early breast cancer on local recurrence and 15-year survival: an overview of the randomised trials. Lancet 2005; 366: 2087-2106.

4. Demmy TL, Nwogu CE, Yendamuri S. Thoracoscopic chest wall resection: what is its role? Ann Thorac Surg 2010; 89: S2142-2145.

5. Wouters MW, van Geel AN, Nieuwenhuis L et al. Outcome after surgical resections of recurrent chest wall sarcomas. J Clin Oncol 2008; 26: 5113-5118.

6. Pignol JP, Caudrelier JM, Crook J et al. Report on the clinical outcomes of permanent breast seed implant for early-stage breast cancers. Int J Radiat Oncol Biol Phys 2015; 93: 614-621.

7. Ji Z, Jiang Y, Tian S et al. The effectiveness and prognostic factors of CT-guided radioactive I-125 seed implantation for the treatment of recurrent head and neck cancer after external beam radiation therapy. Int J Radiat Oncol Biol Phys 2019; 103: 638-645.

8. Wang J, Zhang F, Guo J et al. Expert consensus workshop report: Guideline for three-dimensional printing template-assisted computed tomography-guided (125)I seeds interstitial implantation brachytherapy. J Cancer Res Ther 2017; 13: 607-612.

9. Ji Z, Jiang Y, Guo F et al. Safety and efficacy of CT-guided radioactive iodine- 125 seed implantation assisted by a $3 \mathrm{D}$ printing template for the treatment of thoracic malignancies. J Cancer Res Clin Oncol 2020; 146: 229-236.

10. Sun HT, Jiang YL, Ji Z et al. 3D printing non-coplanar template-assisted 125-iodine seed implantation for thorax movement tumor: individual template design method. Zhonghua Yi Xue Za Zhi 2019; 99: 3699-3702.

11. Qu A, Jiang P, Sun $H$ et al. Efficacy and dosimetry analysis of image-guided radioactive (1)(2)(5)I seed implantation as salvage treatment for pelvic recurrent cervical cancer after external beam radiotherapy. J Gynecol Oncol 2019; 30: e9.

12. Wang L, Wang H, Jiang $Y$ et al. The efficacy and dosimetry analysis of CT-guided (125)I seed implantation assisted with 3D-printing non-co-planar template in locally recurrent rectal cancer. Radiat Oncol 2020; 15: 179.

13. Huang MW, Zhang JG, Zheng L et al. Accuracy evaluation of a 3D-printed individual template for needle guidance in head and neck brachytherapy. J Radiat Res 2016; 57: 662-667.

14. Ji Z, Jiang Y, Guo F et al. Dosimetry verification of radioactive seed implantation for malignant tumors assisted by 3D printing individual templates and CT guidance. Appl Radiat Isot 2017; 124: 68-74.

15. Chen $Y$, Jiang $Y$, Ji Z et al. Dosimetry, efficacy, and safety of three-dimensional printing noncoplanar template-assisted and CT-guided (125)I seed implantation for recurrent retroperitoneal lymphatic metastasis after external beam radiotherapy. Brachytherapy 2020; 19: 380-388.

16. Cox JD, Stetz J, Pajak TF. Toxicity criteria of the Radiation Therapy Oncology Group (RTOG) and the European Organization for Research and Treatment of Cancer (EORTC). Int J Radiat Oncol Biol Phys 1995; 31: 1341-1346.

17. Swarm RA, Paice JA, Anghelescu DL et al. Adult Cancer Pain, Version 3.2019, NCCN Clinical Practice Guidelines in Oncology. J Natl Compr Canc Netw 2019; 17: 977-1007.

18. Watanabe H, Okada M, Kaji Y et al. New response evaluation criteria in solid tumours-revised RECIST guideline (version 1.1). Gan To Kagaku Ryoho 2009; 36: 2495-2501.

19. Putnam JB, Jr. Outcomes of surgery for chest wall sarcomas. Thorac Surg Clin 2010; 20: 535-542.

20. McMillan RR, Sima CS, Moraco NH et al. Recurrence patterns after resection of soft tissue sarcomas of the chest wall. Ann Thorac Surg 2013; 96: 1223-1228.

21. Burt A, Berriochoa J, Korpak A et al. Treatment of chest wall sarcomas: a single-institution experience over 20 years. Am J Clin Oncol 2015; 38: 80-86. 
22. Wan G, Wei Z, Gardi L et al. Brachytherapy needle deflection evaluation and correction. Med Phys 2005; 32: 902-909.

23. Ji Z, Sun H, Jiang Y et al. Comparative study for CT-guided (125)I seed implantation assisted by 3D printing coplanar and non-coplanar template in peripheral lung cancer. J Contemp Brachytherapy 2019; 11: 169-173.

24. Ang QM, Jiang P, Wei $S$ et al. Accuracy and dosimetric parameters comparison of 3D-printed non-coplanar template-assisted computed tomography-guided iodine-125 seed ablative brachytherapy in pelvic lateral recurrence of gynecological carcinomas. J Contemp Brachytherapy 2021; 13: 39-45.

25. Wang LM, Wang H, Jiang Y et al. Dosimetric comparison of computed tomography-guided iodine- 125 seed implantation assisted with and without three-dimensional printing non-coplanar template in locally recurrent rectal cancer: a propensity score matching study. J Contemp Brachytherapy 2021; 13: 18-23.

26. Zhang L, Wang L, Kadeer X et al. Accuracy of a 3-dimensionally printed navigational template for localizing small pulmonary nodules: a noninferiority randomized clinical trial. JAMA Surg 2019; 154: 295-303. 